\title{
Golden Hour Thrombolysis in Acute Ischemic Stroke: The Changing Pattern in South Korea
}

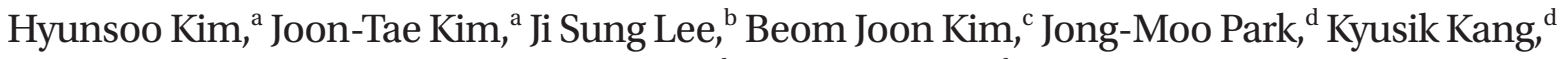 \\ Soo Joo Lee, ${ }^{\mathrm{e}}$ Jae Guk Kim, ${ }^{\mathrm{e}}$ Jae-Kwan Cha, ${ }^{\mathrm{f}}$ Dae-Hyun Kim, ${ }^{\mathrm{f}}$ Tai Hwan Park, ${ }^{\mathrm{g}}$ Sang-Soon Park, ${ }^{\mathrm{g}}$ \\ Kyung Bok Lee, ${ }^{\mathrm{h}}$ Jun Lee, ${ }^{\mathrm{i}}$ Keun-Sik Hong, ${ }^{\mathrm{j}}$ Yong-Jin Cho, ${ }^{\mathrm{j}}$ Hong-Kyun Park, ${ }^{\mathrm{j}}$ Byung-Chul Lee, ${ }^{\mathrm{k}}$ \\ Kyung-Ho Yu, ${ }^{\mathrm{k}}$ Mi Sun Oh, ${ }^{\mathrm{k}}$ Dong-Eog Kim, ${ }^{\mathrm{l}}$ Wi-Sun Ryu, ${ }^{1}$ Jay Chol Choi, ${ }^{\mathrm{m}}$ Jee-Hyun Kwon, ${ }^{\mathrm{n}}$ \\ Wook-Joo Kim, ${ }^{\mathrm{n}}$ Dong-Ick Shin, ${ }^{\mathrm{o}}$ Sung Il Sohn, ${ }^{\mathrm{p}}$ Jeong-Ho Hong, ${ }^{\mathrm{p}}$ Man-Seok Park, ${ }^{\mathrm{a}}$ Kang-Ho Choi, ${ }^{\mathrm{a}}$ \\ Ki-Hyun Cho, ${ }^{a}$ Juneyoung Lee, ${ }^{\mathrm{q}} \mathrm{Hee}-J o o n ~ B a e^{\mathrm{c}}$ \\ aDepartment of Neurology, Chonnam National University Hospital, Chonnam National University Medical School, Gwangju, Korea \\ ${ }^{b}$ Clinical Research Center, Asan Institute for Life Sciences, Asan Medical Center, University of Ulsan College of Medicine, Seoul, Korea \\ 'Department of Neurology, Cerebrovascular Center, Seoul National University Bundang Hospital, Seoul National University College of Medicine, \\ Seongnam, Korea \\ ${ }^{\mathrm{d}}$ Department of Neurology, Nowon Eulji Medical Center, Eulji University, Seoul, Korea \\ 'Department of Neurology, Daejeon Eulji Medical Center, Eulji University, Daejeon, Korea \\ fDepartment of Neurology, Dong-A University Hospital, Dong-A University College of Medicine, Busan, Korea \\ ${ }^{9}$ Department of Neurology, Seoul Medical Center, Seoul, Korea \\ hDepartment of Neurology, Soonchunhyang University Hospital, Soonchunhyang University College of Medicine, Seoul, Korea \\ 'Department of Neurology, Yeungnam University Medical Center, Yeungnam University College of Medicine, Daegu, Korea \\ 'Department of Neurology, Inje University Ilsan Paik Hospital, Inje University College of Medicine, Goyang, Korea \\ kDepartment of Neurology, Hallym University Sacred Heart Hospital, Hallym University College of Medicine, Anyang, Korea \\ 'Department of Neurology, Dongguk University Ilsan Hospital, Dongguk University College of Medicine, Goyang, Korea \\ mDepartment of Neurology, Jeju National University Hospital, Jeju National University School of Medicine, Jeju, Korea

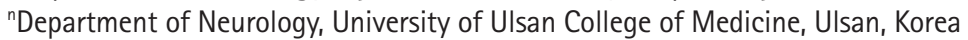 \\ ${ }^{\circ}$ Department of Neurology, Chungbuk National University Hospital, Chungbuk National University College of Medicine, Cheongju, Korea

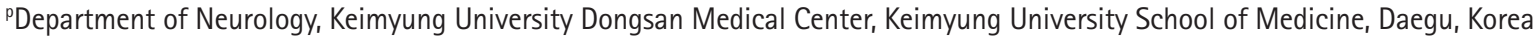 \\ "Department of Biostatistics, Korea University College of Medicine, Seoul, Korea
}

\section{Dear Sir:}

Intravenous tissue plasminogen activator (IV-tPA) is pivotal for the treatment of acute ischemic stroke; however, the benefit of IV-tPA treatment declines rapidly soon after stroke onset. ${ }^{1}$ These results support intensive efforts to reduce both onset-totreatment (OTT) time and door-to-treatment (DTT) time and serve as a basis for establishing an in-hospital and prehospital stroke care system. As these efforts require substantial expenditures of labor and capital, more real-world data are needed to assess the effect of golden hour thrombolysis for acute ischemic stroke. Moreover, understanding the current status and secular changes in IV-tPA treatment will be important to establish an appropriate stroke care system in the future. Therefore, we investigated the effect of golden hour thrombolysis and secular changes in time-to-treatment variables for IV-tPA for acute ischemic stroke by analyzing a prospective registry of 16 stroke centers in South Korea.

This study was based on data from the Clinical Research Collaboration for Stroke in Korea registry of consecutive patients with acute ischemic stroke or transient ischemic attack. From the database, we analyzed the data of patients who were treated with IV-tPA between April 2008 and March 2019. A detailed description of the enrollment process and data collection process is shown in Supplementary Figure 1 and the Supplementary methods. The time metrics, starting from onset or arrival, were defined as follows: (1) onset-to-door (OTD) time was defined as the time from onset (when the patient was last known to be well) to arrival; (2) 0T time was defined as the time from onset to IV-tPA treatment; and (3) DT time was de- 
Table 1. Association of 0 TT time with functional outcomes at 3 months

\begin{tabular}{|c|c|c|c|c|}
\hline Variable & Crude OR (95\% Cl) & $P$ & Adjusted OR (95\% Cl) & $P$ \\
\hline \multicolumn{5}{|l|}{ mRS score of $0-2$ at 3 months } \\
\hline \multicolumn{5}{|l|}{ Binary } \\
\hline $0 \Pi \leq 60 \mathrm{~min}$ & $1.28(0.99-1.64)$ & 0.059 & $1.35(1.01-1.81)$ & 0.043 \\
\hline $0 \pi \mathrm{61-270} \mathrm{min}$ & Reference & & Reference & \\
\hline \multicolumn{5}{|l|}{ Categorical } \\
\hline $0 \Pi \leq 60 \mathrm{~min}$ & $1.59(1.20-2.10)$ & 0.001 & $1.71(1.24-2.35)$ & 0.001 \\
\hline $0 \pi \mathrm{61-120} \mathrm{min}$ & $1.46(1.24-1.71)$ & $<0.001$ & $1.45(1.20-1.74)$ & $<0.001$ \\
\hline $0 \Pi 121-180 \mathrm{~min}$ & $1.18(1.00-1.40)$ & 0.056 & $1.23(1.01-1.50)$ & 0.039 \\
\hline $0 \Pi 181-270 \mathrm{~min}$ & Reference & & Reference & \\
\hline \multicolumn{5}{|l|}{ Continuous } \\
\hline OT, continuous, every $30 \mathrm{~min}$ & $0.92(0.89-0.95)$ & $<0.001$ & $0.92(0.89-0.96)$ & $<0.001$ \\
\hline \multicolumn{5}{|l|}{ mRS distribution (favorable shift) } \\
\hline \multicolumn{5}{|l|}{ Binary } \\
\hline $0 \pi \mathrm{T} \leq 60 \mathrm{~min}$ & $1.30(1.05-1.61)$ & 0.015 & $1.34(1.08-1.67)$ & 0.008 \\
\hline $0 \pi \mathrm{61-270} \mathrm{min}$ & Reference & & Reference & \\
\hline \multicolumn{5}{|l|}{ Categorical } \\
\hline $0 \pi \leq 60 \mathrm{~min}$ & $1.54(1.22-1.95)$ & $<0.001$ & $1.59(1.25-2.03)$ & $<0.001$ \\
\hline 0Tा 61-120 min & $1.36(1.18-1.56)$ & $<0.001$ & $1.32(1.14-1.53)$ & $<0.001$ \\
\hline OT $121-180 \mathrm{~min}$ & $1.10(0.95-1.28)$ & 0.205 & $1.14(0.98-1.32)$ & 0.100 \\
\hline $0 \pi \mathrm{T} 181-270 \mathrm{~min}$ & Reference & & Reference & \\
\hline \multicolumn{5}{|l|}{ Continuous } \\
\hline 0T, continuous, every $30 \mathrm{~min}$ & $0.93(0.90-0.95)$ & $<0.001$ & $0.93(0.91-0.96)$ & $<0.001$ \\
\hline
\end{tabular}

Adjustment variables: age, male sex, initial National Institutes of Health Stroke Scale (NIHSS) score, history of stroke, hypertension, diabetes mellitus, atrial fibrillation, prior statin use, systolic blood pressure, glucose, tissue plasminogen activator dose, and Trial of Org 10172 in Acute Stroke Treatment (TOAST) classification.

OT, onset-to-treatment; $\mathrm{OR}$, odds ratio; $\mathrm{Cl}$, confidence interval; mRS, modified Rankin Scale.
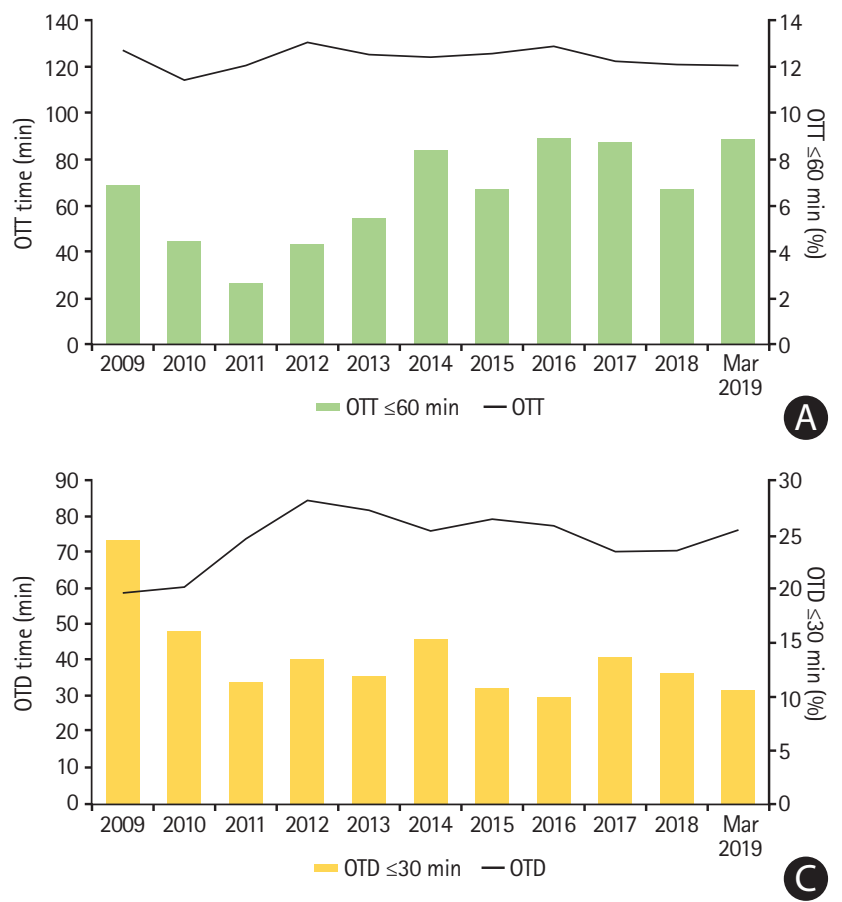

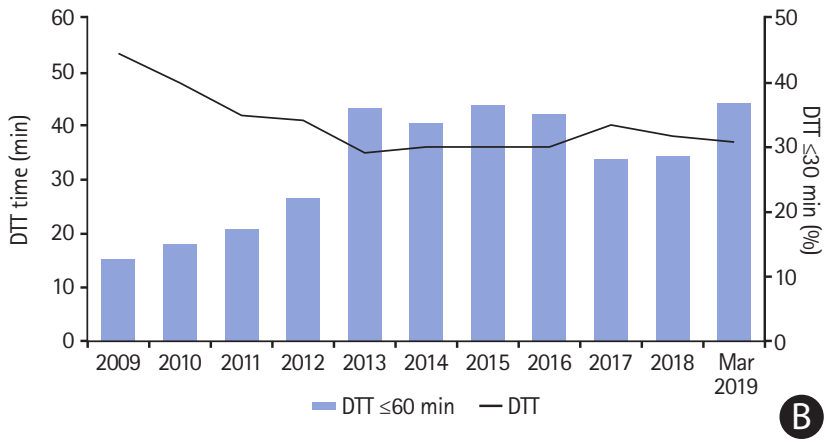

Figure 1. Annual changes in intravenous tissue plasminogen activator treatment times. (A) Onset-to-treatment (OTT) time and the proportions of $0 T$ times $\leq 60$ minutes; (B) door-to-treatment (DTT) time and the proportions of DT times $\leq 30$ minutes; and (C) onset-to-door (OTD) time and the proportions of OTD times $\leq 30$ minutes. 
fined as the time from arrival to IV-tPA treatment. The primary outcome was a good functional outcome at 3 months (a modified Rankin Scale [mRS] score of 0-2). Other outcomes of interest are described in the Supplementary methods.

Multivariable logistic regression models using generalized linear mixed models to account for the effect of hospital (using a random intercept model) were used to explore the relationship between 0T time and the clinical outcome of interest. In addition, we explored the temporal trends in the OT time, DT time, and OTD time by calendar year.

A total of 4,248 patients (mean age $67.6 \pm 12.6$ years; $62 \%$ men) were included. In total, $282(6.6 \%)$ patients had an $0 T$ time between 0 and 60 minutes. The general characteristics of patients according to the four $0 \mathrm{TT}$ windows $(0-60,61-$ $120,121-180$, and 181-270 minutes) are shown in Supplementary Table 1. The associations of $0 \pi T$ windows (as binary, categorical, and continuous variables) with outcomes are shown in Table 1 and Supplementary Table 2. Patients treated within 60 minutes of onset were associated with 35\% higher odds of achieving good outcomes at 3 months than those treated beyond 60 minutes of onset (adjusted odds ratio [aOR], 1.35; 95\% confidence interval [Cl], 1.01 to 1.81$)$. In addition, for every 30-minute delay in treatment, a favorable mRS shift was less likely to occur $(\mathrm{OR}, 0.93 ; 95 \% \mathrm{Cl}, 0.90$ to 0.95) (Supplementary Figure 2).

From April 2008 to March 2019, the proportion of IV-tPA times within the golden hour increased modestly over time, from less than 6.9\% in 2009 to $8.8 \%$ in 2019, with associations of $12 \%$ higher odds for golden hour thrombolysis for every 1-year increase (aOR, $1.12 ; 95 \% \mathrm{Cl}, 1.03$ to $1.21 ; P=0.005)$ (Figure 1 and Supplementary Table 3).

In an analysis of over 4,200 patients treated with IV-tPA from a nationwide multicenter stroke registry in South Korea, golden hour thrombolysis was associated with better functional outcomes at 3 months than later treatment. The risk of death or symptomatic intracerebral hemorrhage was not associated with golden hour thrombolysis. Time to IV-tPA treatment is an important determinant of 90-day functional outcomes in acute ischemic stroke. ${ }^{2}$ In previous studies, golden hour thrombolysis was associated with a good functional outcome at discharge and 3 months. ${ }^{1,3}$ Therefore, our study supports the previous results on the effects of golden hour thrombolysis in real-world practice.

In addition, we found that annual rates of golden hour thrombolysis have substantially increased since 2009. These results seemed to be related to the decrease in DT time or the increasing percentages of DTT time within 30 minutes. Efforts to reduce DTT are the main goal of stroke, and our re- sults support the hypothesis that stroke centers implementing quality improvement programs for in-hospital stroke care improve the workflow of tPA treatment. ${ }^{4-7}$ Unlike the results of DTT reduction, the proportion of patients with OTD $<30$ minutes decreased from that in 2009. This might be an unsolved problem in the stroke system in Korea, and further study is warranted.

This study has several limitations. First, the participating centers did not use uniform guidelines for diagnostic evaluation, patient selection, or IV-tPA treatment workflows. Additionally, detailed hospital factors such as door-to-imaging time were not considered in the analyses. Third, we did not include patients who underwent endovascular thrombectomy. As they might have increased stroke severity, this exclusion could have affected the results. Fourth, our statistical adjustments for patient differences may have been incomplete because of residual or unmeasured confounding variables.

In conclusion, our results show that golden hour thrombolysis could improve the chances of a good outcome at 3 months. Although the data supporting the improvement in in-hospital delay for IV-tPA treatment are clear, the results also suggest that additional efforts to implement more advanced stroke care systems are warranted to further improve acute stroke care in South Korea.

\section{Supplementary materials}

Supplementary materials related to this article can be found online at https://doi.org/10.5853/jos.2020.04658.

\section{References}

1. Kim JT, Fonarow GC, Smith EE, Reeves MJ, Navalkele DD, Grotta JC, et al. Treatment with tissue plasminogen activator in the golden hour and the shape of the 4.5-hour time-benefit curve in the national United States get with the guidelines-stroke population. Circulation 2017;135:128-139.

2. Emberson J, Lees KR, Lyden $P$, Blackwell L, Albers $G$, Bluhmki $E$, et al. Effect of treatment delay, age, and stroke severity on the effects of intravenous thrombolysis with alteplase for acute ischaemic stroke: a meta-analysis of individual patient data from randomised trials. Lancet 2014;384:1929-1935.

3. Tsivgoulis $G$, Katsanos AH, Kadlecová P, Czlonkowska A, Kobayashi $A$, Brozman $M$, et al. Intravenous thrombolysis for ischemic stroke in the golden hour: propensity-matched analysis from the SITS-EAST registry. J Neurol 2017;264:912920.

4. Fonarow GC, Zhao X, Smith EE, Saver JL, Reeves MJ, Bhatt 
$D L$, et al. Door-to-needle times for tissue plasminogen activator administration and clinical outcomes in acute ischemic stroke before and after a quality improvement initiative. JAMA 2014;311:1632-1640.

5. Kamal N, Holodinsky JK, Stephenson C, Kashayp D, Demchuk AM, Hill MD, et al. Improving door-to-needle times for acute ischemic stroke: effect of rapid patient registration, moving directly to computed tomography, and giving alteplase at the computed tomography scanner. Circ Cardiovasc Qual Outcomes 2017;10:e003242.

6. Kassardjian CD, Willems JD, Skrabka K, Nisenbaum R, Barnaby J, Kostyrko $P$, et al. In-patient code stroke: a quality improvement strategy to overcome knowledge-to-action gaps in response time. Stroke 2017;48:2176-2183.

7. Heo JH, Kim YD, Nam HS, Hong KS, Ahn SH, Cho HJ, et al. A computerized in-hospital alert system for thrombolysis in acute stroke. Stroke 2010;41:1978-1983.
Correspondence: Joon-Tae Kim

Department of Neurology, Chonnam National University Hospital, Chonnam National University Medical School, 42 Jebong-ro, Dong-gu, Gwangju 61469, Korea

Tel: +82-62-220-6180

Fax: +82-62-228-3461

E-mail: alldelight2@jnu.ac.kr https://orcid.org/0000-0003-4028-8339

Co-correspondence: Hee-Joon Bae

Department of Neurology, Cerebrovascular Center, Seoul National University Bundang Hospital, Seoul National University College of Medicine, 82 Gumi-ro 173beon-gil, Bundang-gu, Seongnam 13620, Korea

Tel: +82-31-787-7467

Fax: +82-31-787-4059

E-mail:braindoc@snu.ac.kr

https://orcid.org/0000-0003-0051-1997

Received: November 23, 2020

Revised: January 13, 2021

Accepted: January 14, 2021

This study was supported by a grant (BCRI20020) from Chonnam National University Hospital Biomedical Research Institute. This study was supported by funding (2020ER620200\#) from the Research of Korea Centers for Disease Control and Prevention.

The authors have no financial conflicts of interest. 


\section{Supplementary methods}

\section{Data collection}

Demographic, clinical, imaging, and laboratory data were prospectively collected. Baseline data, including National Institutes of Health Stroke Scale (NIHSS) scores, were collected from all patients, and the stroke subtypes were classified according to the Trial of Org 10172 in Acute Stroke Treatment (TOAST) criteria after complete diagnostic profiling. The following data were directly obtained from the registry database: (1) demographics, (2) medical history, (3) medication, (4) stroke characteristics and acute treatment, (5) laboratory data, and (6) in-hospital treatment data. For continuous variables, the data were imputed to the median values if $<5 \%$ of the values were missing.

\section{Outcome measures}

The other outcomes of interest were the distribution of functional outcomes according to the 3-month modified Rankin Scale (mRS) score, an excellent functional outcome at 3 months (a mRS score of 0-1), symptomatic intracerebral hemorrhage (SICH) within 3 months, and death within 3 months. $\mathrm{SICH}$ was defined according to the Safe Implementation of Thrombolysis in Stroke-Monitoring Study criteria as a worsening of neurological status (an increase in NIHSS score of 4 or more) with the appearance of new parenchymal hemorrhage (type 2) on brain imaging that was sufficient to cause neurological deterioration.

\section{Statistical analysis}

The following parameters had missing data that were imputed to the median values: onset-to-treatment (0TT) time $(0.6 \%)$, door-to-treatment (DTT) time (0.6\%), body mass index (2.2\%), creatinine $(0.1 \%)$, hemoglobin $(0.1 \%)$, white blood cell (WBC) count $(0.1 \%)$, and initial random glucose $(0.7 \%)$.

The baseline characteristics, workflow time metrics, and outcomes were compared among patients treated in the $0 T$ windows of $0-60,61-120,121-180$, and 181-270 minutes, and between patients treated within and beyond the golden hour (0T window of 0-60 minutes). Multivariable logistic regression models using generalized linear mixed models to account for the effect of hospital (using a random intercept model) were used to explore the relationship between 0T and the clinical outcome of interest. The adjusted models were controlled for predetermined variables with clinically relevant associations with the outcome variables: age, male sex, initial NIHSS score, history of stroke, hypertension, diabetes mellitus, atrial fibrillation, pre-stroke statin use, systolic blood pressure, glucose, TOAST subtype, tissue plasminogen activator dose, and workflow time variables (OT, DT, and onset-to-door [OTD] times). In addition, we explored the temporal trends in the 0T time (and proportion of $0 T$ times $\leq 30$ minutes), DTT time (and proportion of DT times $\leq 30$ minutes), and OTD time (and proportions of OTD times $\leq 30$ minutes) by calendar year.

$P$-values $<0.05$ were considered statistically significant. Odds ratios and $95 \%$ confidence intervals were calculated. Statistical analysis was performed using $R$ version 3.2 (R Foundation for Statistical Computing, Vienna, Austria). 
Supplementary Table 1. General patient characteristics according to the time to IV-tPA treatment

\begin{tabular}{|c|c|c|c|c|c|c|c|}
\hline Characteristic & $0 \pi \leq 60 \mathrm{~min}$ & 0Tा 61-120 min & OTT 121-180 min & OT 181-270 min & $P$ & $0 \pi>60 \mathrm{~min}$ & $P$ \\
\hline Number & 282 & 1,764 & 1,301 & 901 & & 3,966 & \\
\hline Age (yr) & $66.2 \pm 13.0$ & $66.7 \pm 12.7$ & $68.3 \pm 12.6$ & $68.8 \pm 12.3$ & $<0.001$ & $67.7 \pm 12.6$ & 0.046 \\
\hline Male sex & $178(63.1)$ & $1,122(63.6)$ & $774(59.5)$ & $560(62.2)$ & 0.135 & $2,456(61.9)$ & 0.704 \\
\hline Pre-mRS score of 0-1 & $242(85.8)$ & $1,552(88.0)$ & $1,115(85.7)$ & 778 (86.3) & 0.271 & 3,445 (86.9) & 0.586 \\
\hline Initial NIHSS & $8(4-14)$ & $7(4-13)$ & $6(4-12)$ & $7(4-12)$ & 0.001 & $7(4-12)$ & 0.016 \\
\hline BMI $\left(\mathrm{kg} / \mathrm{m}^{2}\right)$ & $23.7(3.4)$ & $23.9(3.4)$ & $23.4(3.4)$ & $23.5(3.5)$ & 0.005 & $23.6(3.4)$ & \\
\hline Situation & & & & & $<0.001$ & & 0.007 \\
\hline Wake-up & $5(1.8)$ & $44(2.5)$ & $80(6.1)$ & $74(8.2)$ & & $198(5.0)$ & \\
\hline During sleep & $3(1.1)$ & $20(1.1)$ & $41(3.2)$ & $34(3.8)$ & & $95(2.4)$ & \\
\hline During activity & $233(82.6)$ & $1,452(82.3)$ & $928(71.3)$ & $569(63.2)$ & & $2,949(74.4)$ & \\
\hline Unknown & $41(14.5)$ & $248(14.1)$ & $252(19.4)$ & $224(24.9)$ & & $724(18.3)$ & \\
\hline \multicolumn{8}{|l|}{ Workflow times (min) } \\
\hline OाT & $52(48-56)$ & $90(75-103)$ & $150(135-165)$ & $218(198-240)$ & $<0.001$ & 130 (92-178) & $<0.001$ \\
\hline DTT & $26(20-32)$ & $38(29-50)$ & $42(30-57)$ & $41(30-58)$ & $<0.001$ & $40(29-53)$ & $<0.001$ \\
\hline OTD & $25(18-31)$ & $48(34-63)$ & 105 (85-125) & 173 (148-197) & $<0.001$ & $80(48-132)$ & $<0.001$ \\
\hline TOAST & & & & & 0.054 & & 0.025 \\
\hline LAA & $61(21.6)$ & $487(27.6)$ & 391 (30.1) & 278 (30.9) & & $1,156(29.1)$ & \\
\hline SVO & $27(9.6)$ & $219(12.4)$ & $155(11.9)$ & $101(11.2)$ & & 475 (12.0) & \\
\hline CE & $102(36.2)$ & 568 (32.2) & $397(30.5)$ & $258(28.6)$ & & $1,223(30.8)$ & \\
\hline $\mathrm{OE}$ & $7(2.5)$ & $36(2.0)$ & $23(1.8)$ & $11(1.2)$ & & $70(1.8)$ & \\
\hline UD & $85(30.1)$ & $454(25.7)$ & $335(25.7)$ & $253(28.1)$ & & $1,042(26.3)$ & \\
\hline Previous TIA & $4(1.4)$ & $34(1.9)$ & $23(1.8)$ & $11(1.2)$ & 0.581 & $68(1.7)$ & $>0.999$ \\
\hline Previous stroke & 45 (16.0) & $262(14.9)$ & 239 (18.4) & 139 (15.4) & 0.063 & 640 (16.1) & $>0.999$ \\
\hline History of CAD & $32(11.3)$ & 174 (9.9) & $138(10.6)$ & $87(9.7)$ & 0.767 & $399(10.1)$ & 0.475 \\
\hline History of PAD & $0(0.0)$ & $12(0.7)$ & $9(0.7)$ & $0(0.0)$ & 0.022 & $21(0.5)$ & 0.395 \\
\hline HTN & 164 (58.2) & $1,126(63.8)$ & 867 (66.6) & $604(67.0)$ & 0.018 & $2,597(65.5)$ & 0.014 \\
\hline DM & $61(21.6)$ & $460(26.1)$ & $368(28.3)$ & $276(30.6)$ & 0.009 & 1,104 (27.8) & 0.027 \\
\hline Dyslipidemia & $62(22.0)$ & $517(29.3)$ & $340(26.1)$ & $253(28.1)$ & 0.036 & $1,110(28.0)$ & 0.032 \\
\hline Recent smoking & $84(29.8)$ & $537(30.4)$ & $390(30.0)$ & $265(29.4)$ & 0.957 & $1,192(30.1)$ & 0.947 \\
\hline Atrial fibrillation & $97(34.4)$ & $578(32.8)$ & $380(29.2)$ & $268(29.7)$ & 0.084 & $1,226(30.9)$ & 0.231 \\
\hline Prior antiplatelet & $82(29.1)$ & $493(27.9)$ & $377(29.0)$ & $232(25.7)$ & 0.389 & 1,102 (27.8) & 0.631 \\
\hline Prior anticoagulation & $11(3.9)$ & $53(3.0)$ & $57(4.4)$ & $20(2.2)$ & 0.032 & $130(3.3)$ & 0.604 \\
\hline Prior antihypertensive & $131(46.5)$ & $837(47.4)$ & $686(52.7)$ & $457(50.7)$ & 0.019 & $1,980(49.9)$ & 0.268 \\
\hline Prior statin & $54(19.1)$ & $317(18.0)$ & $210(16.1)$ & $162(18.0)$ & 0.456 & $689(17.4)$ & 0.465 \\
\hline Prior antidiabetic & $48(17.0)$ & $328(18.6)$ & $261(20.1)$ & $196(21.8)$ & 0.161 & 785 (19.8) & 0.278 \\
\hline Multiterritory lesions & $33(11.7)$ & $225(12.8)$ & $192(14.8)$ & $148(16.4)$ & 0.037 & $565(14.2)$ & 0.250 \\
\hline IV-tPA dose & & & & & 0.043 & & 0.470 \\
\hline $0.6 \mathrm{mg} / \mathrm{kg}$ & $57(20.2)$ & $407(23.1)$ & $281(21.6)$ & $237(26.3)$ & & $925(23.3)$ & \\
\hline $0.9 \mathrm{mg} / \mathrm{kg}$ & 225 (79.8) & $1,357(76.9)$ & $1,020(78.4)$ & $664(73.7)$ & & $3,041(76.7)$ & \\
\hline \multicolumn{8}{|l|}{ Laboratory finding } \\
\hline WBC $\left(10^{3} / \mu \mathrm{L}\right)$ & $8.05 \pm 2.76$ & $8.03 \pm 2.78$ & $8.32 \pm 3.01$ & $8.67 \pm 3.23$ & $<0.001$ & $8.27 \pm 2.97$ & 0.220 \\
\hline $\mathrm{Hb}(\mathrm{g} / \mathrm{dL})$ & $13.8 \pm 1.9$ & $13.9 \pm 1.9$ & $13.7 \pm 1.9$ & $13.6 \pm 1.9$ & 0.003 & $13.8 \pm 1.9$ & 0.611 \\
\hline Platelets $\left(10^{3} / \mu \mathrm{L}\right)$ & $220.4 \pm 67.1$ & $224.2 \pm 68.3$ & $227.4 \pm 67.6$ & $224.0 \pm 69.2$ & 0.335 & $225.2 \pm 68.3$ & 0.252 \\
\hline Glucose (mg/dL) & $132.3 \pm 44.7$ & $138.0 \pm 51.3$ & $144.8 \pm 60.0$ & $147.5 \pm 58.8$ & $<0.001$ & $142.4 \pm 56.1$ & $<0.002$ \\
\hline LDL-C (mg/dL) & $106.1 \pm 34.3$ & $110.1 \pm 35.1$ & $110.1 \pm 36.5$ & $108.4 \pm 35.9$ & 0.234 & $109.7 \pm 35.7$ & 0.103 \\
\hline PT (INR) & $1.04 \pm 0.17$ & $1.03 \pm 0.14$ & $1.04 \pm 0.29$ & $1.03 \pm 0.13$ & 0.266 & $1.03 \pm 0.20$ & 0.496 \\
\hline SBP $(m m ~ H g)$ & $147.0 \pm 25.4$ & $152.6 \pm 28.5$ & $149.8 \pm 27.3$ & $148.8 \pm 26.9$ & $<0.001$ & $150.8 \pm 27.8$ & 0.015 \\
\hline
\end{tabular}

Values are presented as mean \pm standard deviation, number $(\%)$, or median (interquartile range). $P$-values are from Pearson's chi-square test, Fisher's exact test, analysis of variance (ANOVA), or a Kruskal-Wallis test, where appropriate.

IV-tPA, intravenous tissue plasminogen activator; OT, onset-to-treatment; mRS, modified Rankin Scale; NIHSS, National Institutes of Health Stroke Scale; BMI, body mass index; DT, door-to-treatment; OTD, onset-to-door; TOAST, Trial of Org 10172 in Acute Stroke Treatment; LAA, large artery atherosclerosis; SVO, small vessel occlusion; $C E$, cardioembolism; $O E$, other etiology; UD, undetermined etiology; TIA, transient ischemic attack; $C A D$, coronary artery disease; PAD, peripheral artery disease; HTN, hypertension; DM, diabetes mellitus; WBC, white blood cell; Hb, hemoglobin; LDL-C, low density lipoprotein cholesterol; PT, prothrombin time; INR, international normalization ratio; SBP, systolic blood pressure. 
Supplementary Table 2. Association between 0TT time and functional and safety outcomes

\begin{tabular}{|c|c|c|c|c|}
\hline Variable & Crude OR $(95 \% \mathrm{Cl})$ & $P$ & Adjusted OR $(95 \% \mathrm{Cl})$ & $P$ \\
\hline \multicolumn{5}{|l|}{ mRS $0-1$ at 3 months } \\
\hline \multicolumn{5}{|l|}{ Binary } \\
\hline $0 \Pi \leq 60 \mathrm{~min}$ & $1.24(0.97-1.58)$ & 0.085 & $1.24(0.94-1.63)$ & 0.123 \\
\hline 0Tा 61-270 min & Reference & & Reference & \\
\hline \multicolumn{5}{|l|}{ Categorical } \\
\hline $0 \pi \leq 60 \mathrm{~min}$ & $1.43(1.09-1.87)$ & 0.009 & $1.42(1.05-1.92)$ & 0.025 \\
\hline 0Tा 61-120 min & $1.30(1.11-1.53)$ & 0.002 & $1.25(1.04-1.51)$ & 0.016 \\
\hline 0Tा $121-180 \mathrm{~min}$ & $1.09(0.92-1.30)$ & 0.334 & $1.09(0.90-1.33)$ & 0.364 \\
\hline OTा $181-270 \mathrm{~min}$ & Reference & & Reference & \\
\hline \multicolumn{5}{|l|}{ Continuous } \\
\hline $0 \Pi$, continuous, for every $30-\mathrm{min}$ increase & $0.94(0.91-0.97)$ & $<0.0001$ & $0.94(0.91-0.98)$ & 0.001 \\
\hline \multicolumn{5}{|l|}{ Death } \\
\hline \multicolumn{5}{|l|}{ Binary } \\
\hline $0 \pi \leq 60 \mathrm{~min}$ & $0.87(0.56-1.36)$ & 0.547 & $0.81(0.50-1.30)$ & 0.376 \\
\hline 0Tा 61-270 min & Reference & & Reference & \\
\hline \multicolumn{5}{|l|}{ Categorical } \\
\hline $0 \Pi \leq 60 \mathrm{~min}$ & $0.74(0.46-1.18)$ & 0.207 & $0.67(0.40-1.13)$ & 0.136 \\
\hline 0Tा 61-120 min & $0.75(0.58-0.99)$ & 0.040 & $0.77(0.57-1.04)$ & 0.090 \\
\hline OT $121-180 \mathrm{~min}$ & $0.86(0.65-1.14)$ & 0.285 & $0.82(0.60-1.12)$ & 0.216 \\
\hline OTा $181-270 \mathrm{~min}$ & Reference & & Reference & \\
\hline \multicolumn{5}{|l|}{ Continuous } \\
\hline $0 \Pi$, continuous, for every 30 -min increase & $1.07(1.01-1.12)$ & 0.018 & $1.07(1.01-1.13)$ & 0.030 \\
\hline \multicolumn{5}{|l|}{$\mathrm{SICH}$} \\
\hline \multicolumn{5}{|l|}{ Binary } \\
\hline $0 \pi \leq 60 \mathrm{~min}$ & $0.87(0.35-2.15)$ & 0.757 & $0.87(0.35-2.18)$ & 0.761 \\
\hline 0Tा 61-270 min & Reference & & Reference & \\
\hline \multicolumn{5}{|l|}{ Categorical } \\
\hline $0 \Pi \leq 60 \mathrm{~min}$ & $1.23(0.44-3.49)$ & 0.693 & $1.22(0.42-3.48)$ & 0.716 \\
\hline 0Tा 61-120 min & $1.63(0.87-3.05)$ & 0.130 & $1.55(0.82-2.93)$ & 0.180 \\
\hline 0Tा $121-180 \mathrm{~min}$ & $1.45(0.74-2.82)$ & 0.277 & $1.47(0.75-2.88)$ & 0.267 \\
\hline 0Tा $181-270 \mathrm{~min}$ & Reference & & Reference & \\
\hline \multicolumn{5}{|l|}{ Continuous } \\
\hline $0 \Pi$, continuous, for every 30 -min increase & $0.91(0.81-1.02)$ & 0.101 & $0.92(0.81-1.03)$ & 0.144 \\
\hline
\end{tabular}

Adjustment variables: age, male sex, initial National Institutes of Health Stroke Scale score, history of stroke, hypertension, diabetes mellitus, atrial fibrillation, prior statin use, systolic blood pressure, glucose, tissue plasminogen activator dose, and Trial of Org 10172 in Acute Stroke Treatment (TOAST) classification. $\mathrm{OTT}$, onset-to-treatment; $\mathrm{OR}$, odds ratio; $\mathrm{Cl}$, confidence interval; $\mathrm{mRS}$, modified Rankin Scale; $\mathrm{SICH}$, symptomatic intracerebral hemorrhage. 
Supplementary Table 3. Annual trend in time-to-treatment variables

\begin{tabular}{|c|c|c|c|c|c|c|c|}
\hline Year & No. & OTा (min) & $\mathrm{DTT}$ (min) & OTD (min) & $\mathrm{DTT} \leq 30 \min (\%)$ & $0 \pi T \leq 60 \mathrm{~min}(\%)$ & OTD $\leq 30 \min (\%)$ \\
\hline 2009 & 102 & 126.5 (90 to 160$)$ & 53.5 (40 to 75$)$ & 58.5 (30 to 107.8 ) & 12.75 & 6.86 & 24.51 \\
\hline 2010 & 113 & 114 (85 to 150.5 ) & 48 (35 to 67 ) & 60 (36.5 to 90) & 15.04 & 4.42 & 15.93 \\
\hline 2011 & 349 & 120 (91 to 156$)$ & 42 (34 to 54 ) & 73 (45 to 110 ) & 17.19 & 2.58 & 11.17 \\
\hline 2012 & 417 & 130 (90 to 175$)$ & 41 (31 to 52 ) & 84 (45 to 131$)$ & 22.30 & 4.32 & 13.43 \\
\hline 2013 & 464 & 125 (88 to 175$)$ & 35 (27 to 46$)$ & 81.5 (49 to 133.8 ) & 35.99 & 5.39 & 11.85 \\
\hline 2014 & 565 & 124 (85 to 180$)$ & 36 (28 to 48$)$ & 76 (40.5 to 140$)$ & 33.98 & 8.32 & 15.22 \\
\hline 2015 & 509 & 125 (82 to 174$)$ & 36 (26 to 48$)$ & 79 (43 to 133 ) & 36.54 & 6.68 & 10.81 \\
\hline 2016 & 554 & 128 (82.8 to 187 ) & 36 (25 to 53$)$ & 77 (44 to 137.5 ) & 35.20 & 8.84 & 9.93 \\
\hline 2017 & 504 & 122 (86 to 181.5 ) & 40 (29 to 56$)$ & 70 (40 to 124$)$ & 28.17 & 8.73 & 13.49 \\
\hline 2018 & 477 & 121 (85 to 170 ) & 38 (29 to 53 ) & 70 (40 to 124$)$ & 28.51 & 6.71 & 12.16 \\
\hline Mar 2019 & 125 & 120 (85.5 to 178 ) & 37 (27 to 49$)$ & 76 (46 to 124$)$ & 36.80 & 8.80 & 10.40 \\
\hline Per 1 year $(95 \% \mathrm{Cl})$ & & $0.19(-1.12$ to 1.50$)$ & $-0.53(-1.58$ to 0.53$)$ & $0.67(-0.42$ to 1.77$)$ & NA & NA & NA \\
\hline OR (per 1 yr, 95\% Cl) & & NA & NA & NA & 1.09 (0.96 to 1.24$)$ & 1.12 (1.03 to 1.21$)$ & 0.97 (0.93 to 1.01$)$ \\
\hline$P$ & & 0.774 & 0.327 & 0.229 & 0.171 & 0.005 & 0.192 \\
\hline
\end{tabular}

Values are presented as median (interquartile range). $P$-value by generalized estimating equations linear regression or logistic regression models adjusted for age, sex and initial National Institutes of Health Stroke Scale scores.

OT, onset-to-treatment; DT, door-to-treatment; OTD, onset-to-door; $\mathrm{Cl}$, confidence interval; NA, not applicable; OR, odds ratio. 


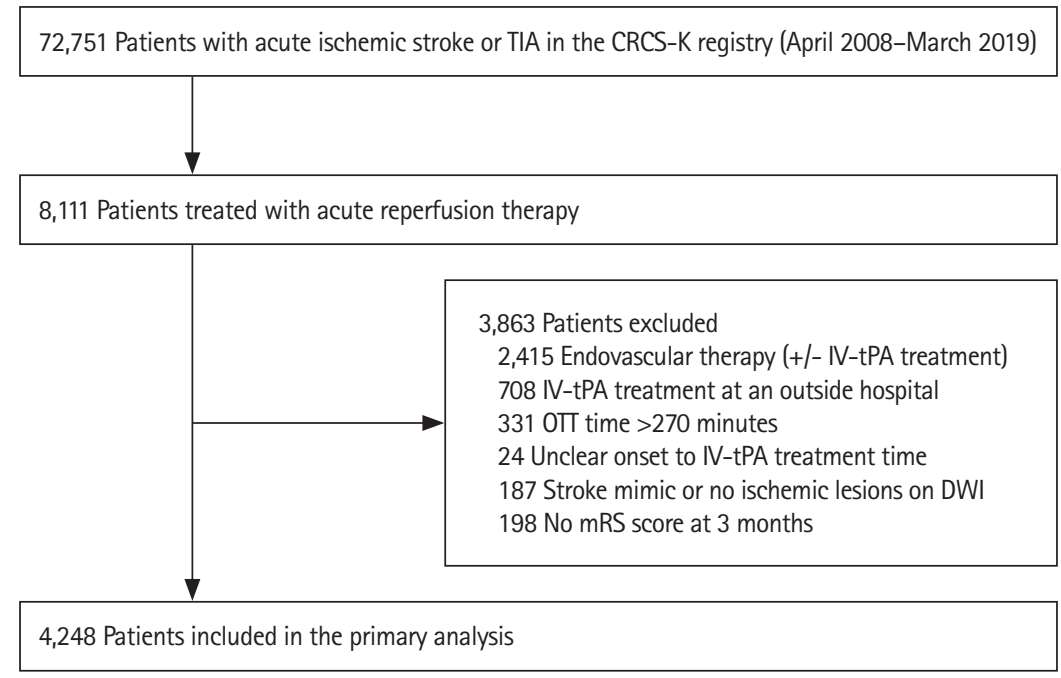

Supplementary Figure 1. Selection of the study population. TIA, transient ischemic attack; CRCS-K, Clinical Research Collaboration for Stroke in Korea; IVtPA, intravenous tissue plasminogen activator; OTT, onset-to-treatment; DWI, diffusion weighted imaging; mRS, modified Rankin Scale.
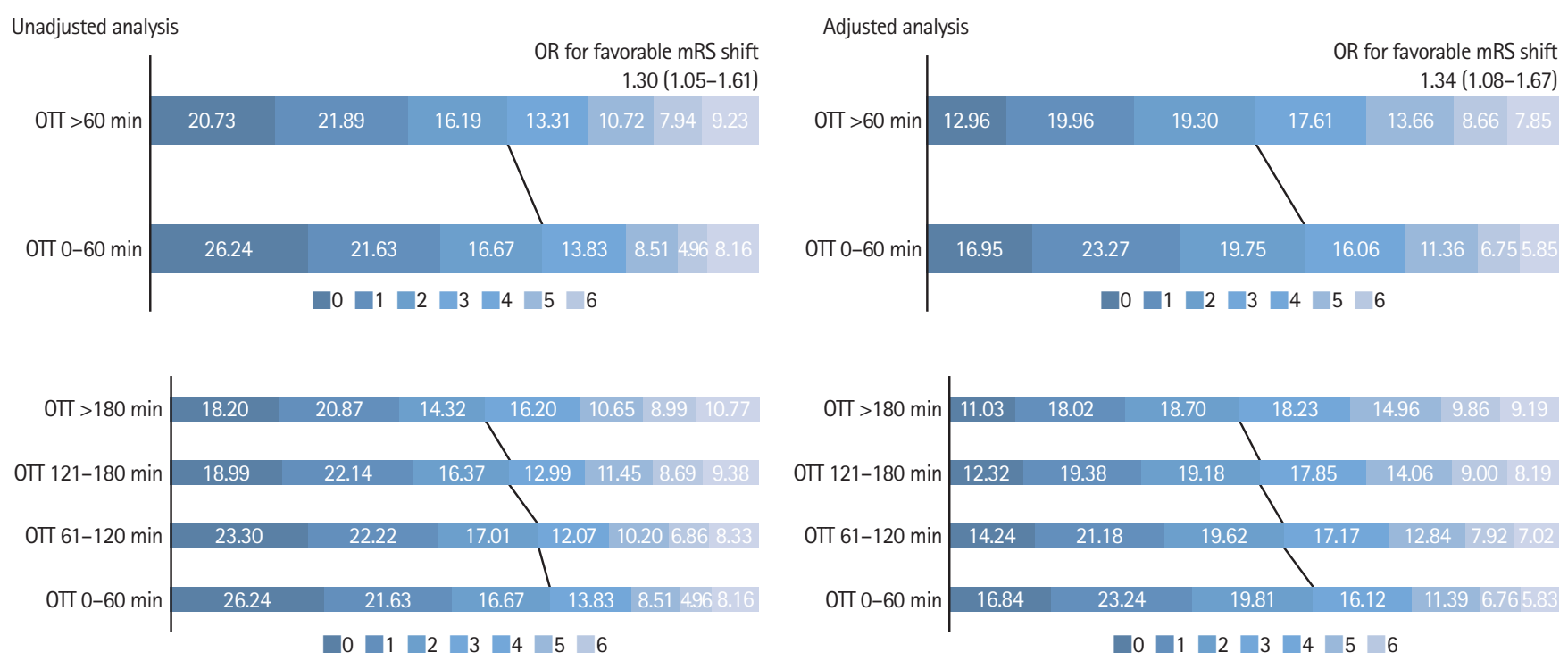

A

Supplementary Figure 2. Unadjusted (A) and adjusted (B) modified Rankin Scale (mRS) score distributions according to onset-to-treatment (OTT) time. OR, odds ratio. 